\title{
Colon capsule for panendoscopy: a narrow window of opportunity
}

\section{(ㄷ)(1) $\odot$}

\author{
Authors \\ Emanuele Rondonotti ${ }^{1}$, Marco Pennazio ${ }^{2}$ \\ Institutions \\ 1 Gastroenterology Unit, Valduce Hospital, Como, Italy \\ 2 University Division of Gastroenterology, City of Health \\ and Science University Hospital, University of Turin, \\ Turin, Italy
}

\section{Bibliography}

Endosc Int Open 2021; 09: E1860-E1862

DOI $10.1055 / a-1548-6572$

ISSN 2364-3722

(C) 2021. The Author(s).

This is an open access article published by Thieme under the terms of the Creative
Commons Attribution-NonDerivative-NonCommercial License, permitting copying and reproduction so long as the original work is given appropriate credit. Contents may not be used for commercial purposes, or adapted, remixed, transformed or built upon. (https://creativecommons.org/licenses/by-nc-nd/4.0/)

Georg Thieme Verlag KG, Rüdigerstraße 14,

70469 Stuttgart, Germany

Corresponding author

Emanuele Rondonotti MD, PhD, Gastroenterology Unit, Valduce Hospital, Via Dante 10, 22100, Como, Italy

Fax: +0039031324150

ema.rondo@gmail.com
The first capsule endoscopy system available in the market was named M2A (M2A Capsule, Givenlmaging Ltd, Yokneam, Israel) [1]. This acronym reveals the philosophy behind this technology: the implementation of a miniaturized system allowing to endoscopically evaluate the entire digestive system, ideally from the mouth to the anus (which is what M2A stands for).

The technical barriers that had initially prevented the exploration of the entire surface of the digestive system have been removed over time through the development of batteries with longer life, the optimization of power consumption (e. g., through the adaptive frame rate) and the increase in the number of cameras and in the angle of view (to cover the entire mucosal surface of wider lumen organs). The study by Vuik et al. [2] in the current issue of Endoscopy International Open, as well as several other publications, [3] confirm that the endoscopic study of the entire surface of the digestive system appears to be now technically possible.

However, does "technical feasible" also mean "clinically reliable"? Although the very quick small bowel transit time reported in the present study (median small bowel transit time: 47') might negatively impact on small bowel capsule diagnostic yield, the key role of capsule endoscopy in the evaluation of the small bowel mucosa has been confirmed in several studies, and current guidelines endorse the use of this device as firstline examination in many clinical scenarios [4]. The clinical reliability concerns, therefore, are related mostly to the evaluation of the colonic, esophageal, and gastric mucosa, which would be the potential added value of panenteroscopy.

In standard colonoscopy, there is a well-documented correlation between the degree of cleanliness and diagnostic per- formance [5]. This is likely to have even greater relevance in the field of wireless endoscopy, where the capsule is purely passive (e. g., it cannot wash or remove debris) and bowel preparation also plays a key role in promoting device propulsion. In the paper from Vuik et al. [2] the rate of patients with adequate colon cleansing (76.6\%) was below the $\geq 90 \%$ minimum standard recommended for optical colonoscopy [6], although involved subjects were healthy and highly compliant with the preparation schedule $(93.6 \%$ of them took the entire bowel preparation). Although this may reflect the specific preparation schedule adopted in the study, a recent systematic review [3] showed that the rate of patients with adequate colon cleansing was highly variable, but $\geq 90 \%$ in only $54 \%$ of studies, even when different schedules were used. Furthermore, although no formal standard quality measure exists, the rate of patients with adequate cleansing of the whole gastrointestinal tract $(52.8 \%)$ is far from being acceptable. Finally, as far as the completion rate for colon evaluation is concerned, the $51 \%$ rate reported by Vuik et al. [2] is similar to those reported in other publications [7-9] and far below the recommended threshold of $90 \%$ [6].

Although relevant gastric findings, missed by previous traditional gastroscopy, were reported in patients undergoing small bowel capsule endoscopy [10], the parameter for assessing the efficacy of panenteroscopy in evaluation of gastric mucosa (observation of more than $90 \%$ of the mucosa) appears to be arbitrary, contrived, and very difficult to measure. In the absence of a traditional examination as comparator, no clinically relevant conclusions can be drawn about the diagnostic yield in the stomach of such a non-steerable capsule. In addition, because 
the capsule was ingested without a dedicated protocol for esophageal inspection [11], the $Z$ line was identified in fewer than $50 \%$ of the subjects included in the study, underscoring that capsule endoscopy, when administered as a panenteroscopy tool, cannot represent a viable alternative to regular gastroscopy.

Patient acceptability has always been emphasized as one of the major advantages of capsule endoscopy over more invasive and less tolerated procedures, such as gastroscopy and colonoscopy. However, Vuik et al. [2] demonstrated that moving from theory (pre-procedural questionnaire) to practice (postprocedural questionnaire), patient expectations are somewhat disregarded. In line with these results, in the TOPAZ study [12] more subjects who underwent both procedures under evaluation unexpectedly preferred traditional colonoscopy over colon capsule endoscopy. Once again, the major determinant behind this preference was the burden related to colon capsule endoscopy bowel preparation.

Taking into account all these issues, one might wonder if panenteric capsule endoscopy evaluation is a journey worth undertaking and a goal worth pursuing. Taken individually, each traditional endoscopic examination for evaluation of esophagus, stomach and colon seems to be more reliable than panenteroscopy. However, the latter offers the unique opportunity to combine evaluation of several segments of the digestive tract at the same time, in a single examination. This becomes particularly relevant when the "timing of the procedure" or "disease extent" are key factors. Indeed, there is growing evidence supporting the use of a panenteroscopic approach in patients with digestive bleeding, in whom an upper digestive tract lesion has been excluded $[13,14]$. Initial data suggest that in these patients, a timely panenteric evaluation has relevant clinical (increased rate of diagnosis, crucial information for therapeutic work-up) as well as organizational/economic consequences (reduction of the number of unnecessary examinations and hospitalization time). Similarly, for staging and surveillance in patients with Crohn's disease, especially in the pediatric setting, panenteroscopy appears to be a reliable tool for assessing disease mucosal activity and extent, compared to more invasive methods [15-17].

In light of all the pros and cons, with current technology and organizational/practical issues (e.g. the preparation schedule), the window of opportunity for panenteroscopy in everyday clinical practice currently is narrow. However, the most limiting factors appear amenable to significant improvements, and the introduction of automated image analysis systems (e. g. artificial intelligence-based software) [18], which could make the procedure even easier and more reliable, is expected soon. These improvements are needed before implementing use of the capsule for whole-intestine mucosal assessment in clinical practice.

\section{Acknowledgements}

The authors thank Dr. Franco Radaelli for support during the writing and editing process.
Competing interests

Dr. Rondonotti received speaker honoraria from Fujifilm Co, consultancy agreement with Medtronic Co. Dr. Pennazio received speaker honoraria from Olympus Co, Medtronic Co.

\section{References}

[1] Meron GD. The development of the swallowable capsule video capsule (M2A). Gastrointest Endosc 2000; 52: 817-819

[2] Vuik FER, Moen S, Nieuwenburg SAV et al. Applicability of colon capsule endoscopy as panendoscopy: from bowel preparation, transitand rating times to completion rate and patient acceptance. Endoscopy Intern Open 2021; 09: E1852-E1859

[3] Cortegoso Valdivia P, Elosua A, Houdeville C et al. Clinical feasibility of panintestinal (or panenteric) capsule endoscopy: a systematic review. Eur J Gastroenterol Hepatol 2021; 33: 949-955

[4] Pennazio M, Spada C, Eliakim R et al. Small-bowel capsule endoscopy and device-assisted enteroscopy for diagnosis and treatment of small-bowel disorders: European Society of Gastrointestinal Endoscopy (ESGE) Clinical Guideline. Endoscopy 2015; 47: 352-376

[5] Kluge MA, Williams JL, Wu CK et al. Inadequate Boston Bowel Preparation Scale scores predict the risk of missed neoplasia on the next colonoscopy. Gastrointest Endosc 2018; 87: 744-751

[6] Kaminski MF, Thomas-Gibson S, Bugajski M et al. Performance measures for lower gastrointestinal endoscopy: a European Society of Gastrointestinal Endoscopy (ESGE) Quality Improvement Initiative. Endoscopy 2017; 49: 378-397

[7] Carretero Ribon C, Prieto-Frias C, Angos R et al. Pan-enteric capsule for bleeding high risk patients. Can we limit endoscopies? Rev Esp Enferm Dig 2020: doi:10.17235/reed.2020.7196/2020

[8] Yamada K, Nakamura M, Yamamura T et al. Diagnostic yield of colon capsule endoscopy for Crohn's disease lesions in the whole gastrointestinal tract. BMC Gastroenterol 2021; 21: 75

[9] Eliakim R, Yablecovitch D, Lahat A et al. A novel PillCam Crohn's capsule score (Eliakim score) for quantification of mucosal inflammation in Crohn's disease. United European Gastroenterol J 2020; 8: 544-551

[10] Innocenti T, Dragoni G, Roselli ] et al. Non-small-bowel lesions identification by capsule endoscopy: A single centre retrospective study. Clin Res Hepatol Gastroenterol 2021; 45: 101409

[11] Gralnek IM, Rabinovitz R, Afik D et al. A simplified ingestion procedure for esophageal capsule endoscopy: initial evaluation in healthy volunteers. Endoscopy 2006; 38: 913-918

[12] Cash BD, Fleisher MR, Fern S et al. Multicentre, prospective, randomized study comparing the diagnostic yield of colon capsule enoscopy versus CT colonography in a screening population (TOPAZ study). Gut 2020: doi:10.1136/gutjnl-2020-322578

[13] Yung DE, Koulaouzidis A, Douglas S et al. Earlier use of capsule endoscopy in inpatients with melena or severe iron deficiency anemia reduces need for colonoscopy and shortens hospital stay. Endosc Int Open 2018; 6: E1075-E1084

[14] Mussetto A, Arena R, Fuccio L et al. A new panenteric capsule endoscopy-based strategy in patients with melena and a negative upper gastrointestinal endoscopy: a prospective feasibility study. Eur J Gastroenterol Hepatol 2021; 33: 686-690

[15] Tai FWD, Ellul P, Elosua A et al. Panenteric capsule endoscopy identifies proximal bowel disease guiding upstaging and treatment intensification in Crohn's disease: a European multicentre observational cohort study. United European Gastroenterol J 2021; 9: 248-255 
[16] Oliva S, Aloi M, Viola F et al. A treat o target strategy using panenteric capsule endoscopy in pediatric patients with Crohn's disease. Clin Gastroenterol Hepatol 2019; 17: 2060-2067

[17] Bruining DH, Oliva S, Fleisher MR et al. Panenteric capsule endoscopy versus ileocolonoscopy plus magnetic resonance enterography in
Crohn's disease: a multicentre, prospective study. BM] Open Gastroenterol 2020; 7: e000365

[18] Dray X, lakovidis D, Houdeville C et al. Artificial intelligence in small bowel capsule endoscopy - current status, challenges and future promise. J Gastroenterol Hepatol 2021; 36: 12-19 